Research Paper

\title{
AAV-Mig-6 Increase the Efficacy of TAE in VX2 Rabbit Model, Is Associated With JNK Mediated Autophagy
}

\author{
Zixuan $\mathrm{Li}^{1,2}$, Yulong Tian ${ }^{1,2}$, Lianyue $\mathrm{Qu}^{3}$, Jingsong Mao ${ }^{2,4}$, Hongshan Zhong1,2® \\ 1. Department of Interventional Radiology, The First Affiliated Hospital of China Medical University, Shenyang, China \\ 2. Key Laboratory of Diagnostic Imaging and Interventional Radiology of Liaoning Province, Shenyang, China \\ 3. Department of Pharmacy, The First Affiliated Hospital of China Medical University Shenyang, China \\ 4. Department of Radiology, Xiang'an Hospital of Xiamen University, Xiamen, P. R. China \\ $\bowtie$ Corresponding author: Hongshan Zhong Ph.D., Professor, Department of. Radiology, The First Affiliated Hospital of China Medical University, Shenyang, \\ China \\ (1) Ivyspring International Publisher. This is an open access article distributed under the terms of the Creative Commons Attribution (CC BY-NC) license \\ (https://creativecommons.org/licenses/by-nc/4.0/). See http://ivyspring.com/terms for full terms and conditions.
}

Received: 2018.05.22; Accepted: 2018.12.10; Published: 2019.01.29

\begin{abstract}
The characterization of high recurrence rate of $\mathrm{HCC}$ after TAE provides insights into persistent issues surrounding the role of adjunct therapies administered with TAE. As a regulator of the HER family, Mig-6 is down-regulated in HCC and predicts the prognosis of HCC. In this study, we found up-regulation the expression of Mig-6 enhances autophagy in HCC cells. This function of Mig-6 is related to the activation of the JNK pathway. Next AAV-9 encoding Mitogen inducible gene 6 (Mig-6) was delivered into VX2 liver transplant tumor of rabbits by using hepatic artery catheter. Wild-type AAV is not associated with any human or animal disease and has very low immunogenicity. There are over 100 different AAV serotypes that vary in the amino acid sequence of their capsid protein. We also describe a novel combination therapy coupling AAV-Mig-6 and $T A E$ in a rabbit model resulted in a growth rate decrease in tumor compared with TAE alone. Furthermore, we show that the changes of LC3b and p62, as well as the p-JNK were consistent with changes in vitro experiments. These results suggest that Mig-6 efficiently inhibits tumor progression in vivo. Our findings suggest that Mig-6 induced autophagy inhibition may become a necessary target for adjunct therapy in TAE.
\end{abstract}

Key words: Mig-6, TAE, AAV, Autophagy, JNK

\section{Introduction}

Hepatocellular carcinoma (HCC) is the second leading cause of cancer in 2012 alone [1]. Although surgery and liver transplantation are the preferred methods of treating liver cancer, less than $20 \%$ of patients are suitable for surgical treatment. At present, the professional guidelines for the treatment of liver cancer believe that for unresectable advanced liver cancer, transcatheter embolization (TAE) is an effective treatment. Embolic treatment can lead ischemic injury of the tumor, eventually causing tumor infarction. After treatment, hypoxia and a series of molecular changes in the non-infarct area may become a fortress in the protection of primary tumor [2]. So, despite the continuous development of key therapeutic techniques, the efficacy of TAE is still limited. The recurrence of local lesions often requires continuous treatment. Therefore, it is imperative to explore effective combination regimen allows the microenvironment of residual tumor resensitive to the treatment.

Our previous researches focus on study the function of Mig- 6 in cancer. Mig- 6 is a biomarker which is down-regulated in HCC and predicts the prognosis of HCC. Mig-6 is involved in the activity of multiple receptor tyrosine kinase pathways. These pathways have important regulatory roles in tumor microenvironment [3].

Autophagy is an important part of the tumor microenvironment. Although in the occurrence and development of HCC, the role of autophagy is dependent on the context of liver cells. However, once a tumor is established, unbalanced autophagy will contribute to HCC cell survival under various stress conditions and in turn promotes tumor growth [4]. There also a study showed that autophagy can be induced by (Transcatheter Arterial Chemoembo- 
lization) TACE in liver cancer animal mode [5].

The autophagy in tumor is regulated by multiple tyrosine kinase pathways. Therefore, Mig- 6 , which affects these tyrosine kinase pathways, is likely to regulate autophagy in tumor cells and thus affect tumor microenvironment. However, there is no study focus on the relationship between Mig-6 and autophagy.

Mig-6 has been proved to be a tumor suppressor in a variety of tumors. However, whether the restoration of Mig- 6 expression in tumor tissue can play a role in inhibiting tumor growth has not been experimentally confirmed. After TAE, whether the change of this microenvironment in the residual tumor is related to the receptor tyrosine kinase pathway, and whether Mig-6 can affect the microenvironment change has not been studied.

In this study, we use AAV as gene transfection vector. Wild-type AAV is has been previously used in human gene therapy in 92 clinical trials [6]. There are over 100 different AAV serotypes [7] that vary in theamino acid sequence of their capsid protein. However, so far, no AAV serotype has been used to deliver genes for transfection in the liver transplant tumor model. In this study, we injected AAV-9 encoding Mig-6 into the VX2 liver transplant tumors of rabbits by TAE method. This animal model can improve our understanding of microenvironment of tumor after TAE in liver cancers. The purpose of our study was to characterize the role of Mig-6 in inhibiting tumor autophagy which may be exploited to potentiate TAE in vivo.

\section{Materials and methods}

\section{Cell lines}

HepG-2 andSMMC7721 cell lines were obtained from American Type Culture Collection (Manassas, VA).The cells were cultured in DMEM (Biological Industries) containing 10\% fetal calf serum (Biological Industries), $100 \mathrm{IU} / \mathrm{mL}$ penicillin (Sigma, St.Louis, $\mathrm{MO}$ ), and $100 \mathrm{mg} / \mathrm{mL}$ streptomycin (Sigma).Cells were grown on sterile T25 cell culture flask (Corning) and were passaged every $3 \mathrm{~d}$ using $0.25 \%$ trypsin (Keygen Biotech).

\section{Transfection treatment}

The cells were seeded in 6-well plates $24 \mathrm{~h}$ prior to the experiment. Cells were transfected with empty vector (pcDNA3) or Mig-6 overexpression vector (pcDNA3-Mig-6) (both from Takara Biotechnology, Dalian, China) using Lipofectamine ${ }^{\circledR}$ 3000(Thermo Fisher Scientific) according to the manufacturer's protocol. The mRNA and protein levels were assessed $48 \mathrm{~h}$ following transfection.

\section{Quantitative Real-Time PCR (SYBR Green Method)}

Quantitative real-time PCR was performed using SYBR GreenImaster (Roche)in a total volume of $20 \mu \mathrm{L}$ on Light Cycler ${ }^{\circledR} 480 \mathrm{II}\left(\right.$ Roche) as follows: $95^{\circ} \mathrm{Cfor} 5 \mathrm{~min}$, 40 cycles of $95^{\circ} \mathrm{C}$ for $10 \mathrm{~s}, 60^{\circ} \mathrm{Cfor} 20 \mathrm{~s}$. A dissociation step was performed to generate a melting curve to confirm the specificity of the amplification. $\beta$-actin was used as the reference gene. The primer sequences are provided in the Table 1. Experiments were repeated in triplicate.

Table 1.

\begin{tabular}{ll}
\hline Name & Primer sequences \\
\hline Actin forward & 5'-ATAGCACAGCCTGGATAGCAACGTAC-3' \\
Actin reverse & 5'-CACCTTCTACAATGAGCTGCGTGTG-3' \\
Mig-6 forward & 5'-TCTTCCACCGTTGCCAATCT-3' \\
Mig-6 reverse & 5'-TTCGCCTGCCAGGAACATC-3' \\
\hline
\end{tabular}

\section{Western blot analysis}

Total proteins from cell lines and tissues were extracted in a lysis buffer (CAT.78510) (Thermo Fisher Scientific, Rockford, IL) and quantified using Bradford method (CAT.23226) (Thermo Fisher Scientific). Sixty micrograms of protein were separated by SDS-PAGE (10\%). After transferring by Trans-Blo ® Turbo ${ }^{\mathrm{TM}}$ (Bio-Rad, USA), the polyvinylidene fluoride (PVDF) membranes (Millipore, Billerica, MA, USA) were incubated overnight at $4{ }^{\circ} \mathrm{C}$ with the following antibodies Mig- 6 (1: 1000, CAT.11630-1-AP) (protein tech), anti-P62/SQSTM1 (1: 1000,CAT.55274-1-AP)(protein tech), GFP (1:1000,CAT.ab38689) (Abcam), $\beta$-actin (1:2000, CAT.20536-1-AP) (protein tech), anti-phospho-JNK (Thr183/Tyr185) (1:1000, CAT.4668) (Cell Signaling Technology, Danvers, MA), anti-LC3b (1:2000, CAT.NB100-2220) (Novus Biologicals USA), anti-JNK (1:1000, CAT.AJ518) (Beyotime Biotechnology). After incubation with peroxidase-coupled anti-mouse or rabbit IgG (Santa Cruz Biotechnology) at $37^{\circ} \mathrm{C}$ for 2 hours, bound proteins were visualized using ECL (Thermo Fisher Scientific) and detected using Chemidoc ${ }^{\mathrm{TM}} \mathrm{MP}$ Imaging Systerm (Bio-Rad, USA). The relative protein levels were calculated based on $\beta$-actin as the loading control.

\section{Immunohistochemistry}

Immunostaining was performed using the non-biotin amplification complex method (EliVisionTM super, Maixin, Fuzhou, China). The sections were deparaffinized in xylene, rehydrated in graded alcoholseries, and boiled in $0.01 \mathrm{M}$ citrate buffer (pH 6.0) for $2 \mathrm{~min}$ in an autoclave. Endogenous peroxidase activity was blocked using hydrogen 
peroxide $(0.3 \%)$, this was followed by incubation with normal goat serum to reduce nonspecific binding. Tissue sections were incubated with GFP antibody (1:100, ab38689) (Abcam), P-JNK antibody (1:100,81E11) (CST), P62 antibody (1:200, 55274-1-AP) (protein tech), LC3B (1:400, NB100-2220) (Novus). Staining for all primary antibodies was performed at room temperature for $1.5 \mathrm{~h}$. Elivision ${ }^{\mathrm{TM}}$ super HRP (Mouse/Rabbit) IHC Kit was used. After washing, the sections were incubated with DAB-2031 (Maixin), to develop the peroxidase reaction. Counterstaining of the sections was done with hematoxylin, which were then dehydrated in ethanol before mounting. Two independent investigators examined all tumor slides randomly. Five views were examined per slide.

\section{Evaluation of fluorescent LC3 puncta}

LC3 puncta were indicated by mRFP-GFP-LC3 adenovirus (Gene Chem, Shanghai, Chian) Briefly, cells were transfected with mRFP-GFP-LC3 for $48 \mathrm{~h}$ before receiving Transfection treatments. After 24-hours Transfection treatment, cells were observed under a fluorescence microscopy (Nikon). Ten views were examined per slide and the images were acquired.

\section{Animal model}

The protocol for this animal experimentation was approved by the Animal Care and Use Committee of China Medical University. All methods were performed in accordance with the relevant guidelines and regulations. Healthy New Zealand white rabbits weighing $2-2.5 \mathrm{~kg}$, and rabbitVX2 carcinoma cells were used in this study. Briefly, solid tumors for implantation were obtained by injecting VX2 tumor cell suspension into both thigh muscles of a carrier rabbit. Two weeks later, fresh VX2 tumors harvested were cut into $1-\mathrm{mm}^{3}$ blocks, then a rabbit VX2 liver tumor model was induced by inserting 1 piece of tumor into one location in the left lobe of the liver of the rabbits for experiments using a ultrasound-guided percutaneous puncture implantation method. Fourteen days after tumor implantation, a diameter of approximately $6-20 \mathrm{~mm}$ in the liver can be examined. The tumor models were evaluated by magnetic resonance imaging (MRI).

\section{Experimental design}

We choose 5 rabbits as control group and 40 rabbits which tumor diameter of 10-15 mm accept next experiment. The rabbits were divided into four groups. The tumor volume (V) was calculated according to the following equation: $\mathrm{V}=\mathrm{a} \times \mathrm{b}^{2} / 2$, where " $a$ " is the longest diameter and " $b$ " is the shortest diameter of the tumor. Depending on the calculated tumor volume, rabbits with similar-size tumors were distributed evenly to each group. The animal was placed in the Ultrasonic apparatus. AAV-CMV-3FLAG and AAV-CMV-Mig-6-GFP3FLAG were purchased from Gene Chem (Shanghai, Chian). Using ultrasound guidance and an 18-gauge needle, AAV was delivered directly into the tumor (IT) group. In the IT-control group, ten rabbits were treated with AAV-CMV-3FLAG $\left(25 \mu \mathrm{L}\right.$ with $\left.1 \times 10^{11} \mathrm{vg}\right)$ dissolved in $0.2 \mathrm{ml} / \mathrm{kg}$ physiological saline. In IT-AAV-Mig- 6 group ten rabbits were treated with AAV-CMV-Mig-6-GFP-3FLAG $\left(50 \mu \mathrm{L}\right.$ with $\left.1 \times 10^{11} \mathrm{vg}\right)$ dissolved in $0.2 \mathrm{ml} / \mathrm{kg}$ physiological saline. In the TAE group, ten rabbits were treated with iodized oilvia arterial catheterization. In TAE plus AAV-Mig-6 group ten rabbits were treated with AAV-CMV-Mig-6-GFP-3FLAG $\left(100 \mu \mathrm{L}\right.$ with $2 \times 10^{11}$ vg) dissolved in $0.2 \mathrm{ml} / \mathrm{kg}$ physiological salinein addition to iodized oil. All of the rabbits were given intramuscular injection of penicillin for five days after the AAV injection. All the animals were sacrificed on days 21 . The tumor volume was measured and the tumor growth ratio was calculated by comparing the tumor volume obtained before and 21 days after the treatments.

\section{TAE}

Experimental rabbits were fasted for 12 hours on the operation day but were allowed to consume water. Then, rabbits were anesthetized, placed in supine position on a home-made platform, with limbs fixed. The hair was removed in the xiphoid and upper abdomen area, and disinfected with ultraviolet for 30 min. Rabbits were maintained under general anesthesia. Angiography was performed with a C-arm unit Mobile X-Ray Image System ARCADIS Varic (SIEMENS). Selective arteriography of celiac artery was performed by a micro catheter $(2.7 \mathrm{~F} / 2.9 \mathrm{~F}$ TERUMO, Tokyo, Japan). The left hepatic artery was administered by catheterization. After the drug infusion, the catheter was removed and the 6-0 prolene suture was used to suture the femoral artery.

\section{Liver function measurement}

Blood samples were collected from IT group and TAE group before and at 3, 7and 14 day postoperatively. The plasma alanine aminotransferase (ALT) and aspartateamnotransferase (AST) levels were tested using ELISA assay kits purchased from Boster (Wuhan, China), according to the manufacturer's instructions.

\section{Statistical analysis}

SPSS version19.0 for windows was used for analyses. Student's t-test was used to compare differences between groups. p-values were based on the two-sided statistical analysis, and $p<0.05$ was 
considered to indicate a statistically significant difference.

\section{Results}

\section{Up-regulation the expression of Mig-6 inhibits autophagy in HCC cells.}

In the present study, HepG-2 and SMMC7721 cells treated with Mig- 6 plasmid were monitored for autophagy. To determine transfection efficiency, the mRNA and protein level were evaluated using RT-PCR and western blotting, respectively. The Mig-6 expression was increased in the Mig- 6 plasmid groups compared with the empty vector (EV) groups (Fig. 1). To determine whether the up-regulation of Mig-6 is able to induce autophagy, autophagy-associated protein expression levels were evaluated using western blotting (Fig. 2A). The expression levels of microtubule associated protein 1 light chain $3 \beta$ (LC3b), following transfection with Mig-6 plasmid were significantly decreased compared with those of the EV groups, but p62, which is mainly degraded by autophagy pathway, is greatly induced in the Mig-6 plasmid groups. Autophagy inhibitor 3-Methyladenine (3-MA) was used as a positive control in these experiments.

To further examine the impact of Mig- 6 on autophagic flux in two cell lines, we used mRFP-GFP-LC3 fluorescence to detect autophagosomes. As shown in Fig. 2B, C HepG-2 and SMMC7721 cells have higher basal levels of autophagy, Mig-6 plasmid as well as 3-MA weakened flux of autophagy, with a less number of autophagosomes (yellow) and autolysosomes (red) in two cell lines.

\section{Mig-6 inhibits the autophagy of HCC cells by activating the SAPK / JNK pathway}

In recent years, many studies focus on the role of Mig- 6 in the regulation of protein kinase pathways such as mTOR and P38. As a mitogen-induced gene, Mig-6 also regulates the mitogen-activated protein kinase (MAPK) subgroup stress-activated protein kinas (SAPK). Earlier literature confirmed Mig-6 can activate the SAPK/JNK pathway [8].However, the relationship between Mig-6 and JNK has not been reported in the HCC. In the present study, we found that p-JNK increased after Mig-6 up-regulation, and p-JNK decreased when SP600125, a JNK pathway inhibitor, was also added. SP600125 also increase the expression of LC3b, but decrease the expression of p62 in two cell lines (Fig. 3A). To further elucidate the relationship between activation of the JNK pathway and autophagy in HCC cells, we performed autophagic flux assays following Mig-6 transfection. Upregulation of Mig-6 inhibited HCC autophagic flux effectively. The autophagy level of two HCC cells with Mig- 6 overexpression can be markedly increased by exogenous JNK inhibitor SP600125, as evidenced by significantly increased fluorescence points (Fig. 3B, C).The above result shows that inhibition of autophagy by Mig-6 in HCC cells is related to the activation of the JNK pathway.
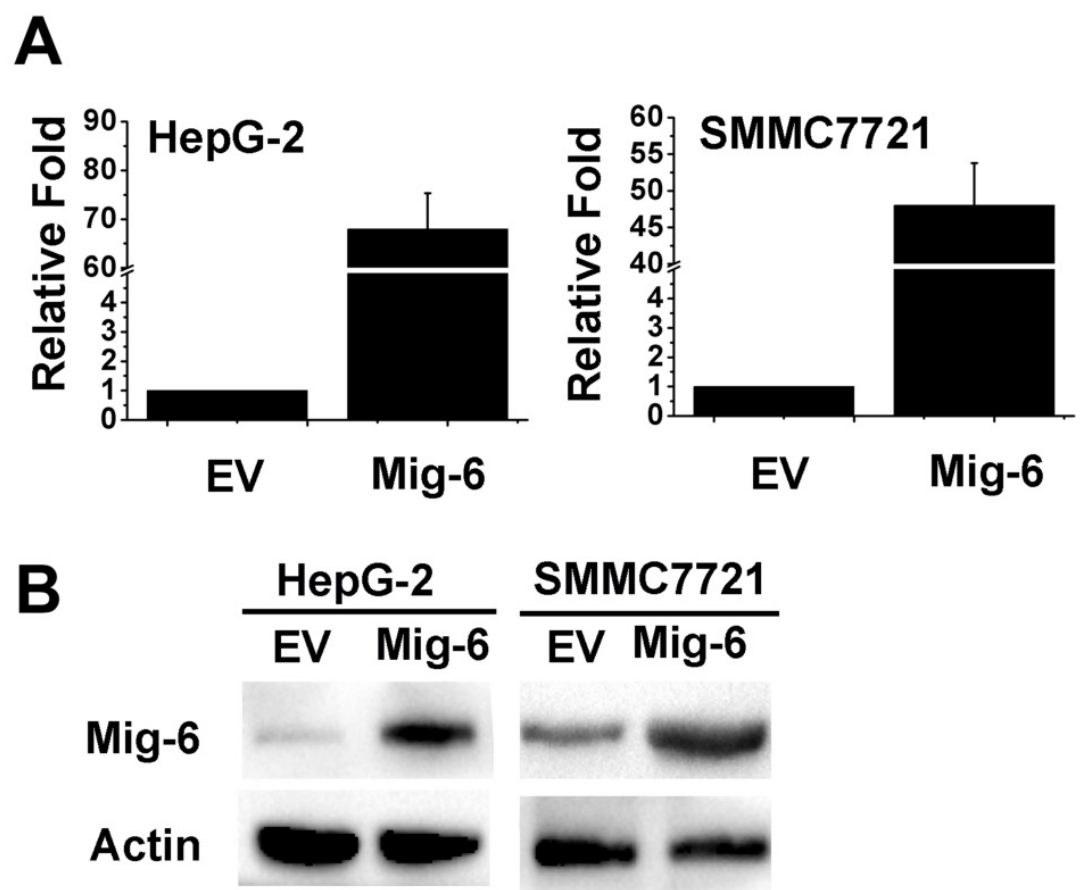

Figure 1. Mig-6 is over-expression in liver cancer cells. (A) Transfected efficiency as assessed by real-time PCR $48 \mathrm{~h}$ after transfection in HepG-2 and SMMC7721 cell lines. (B) Western bloting analysis of transfection efficiency of Mig-6 plasmid in HepG-2 and SMMC7721 cell lines. 
A

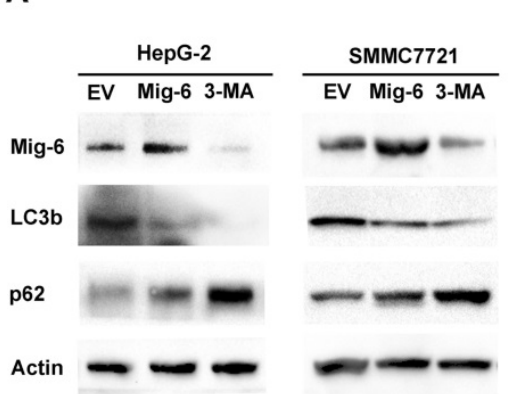

C

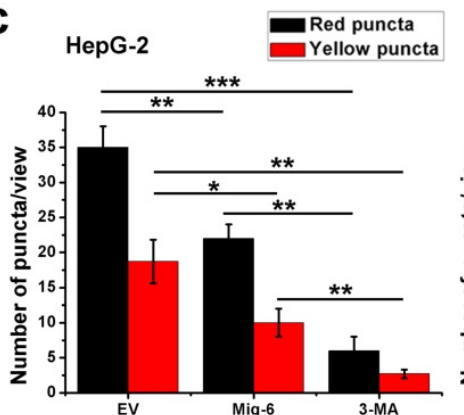

SMMC7721

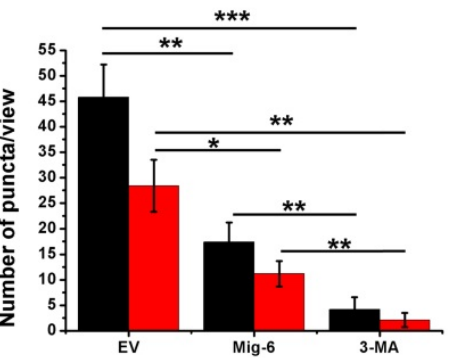

B

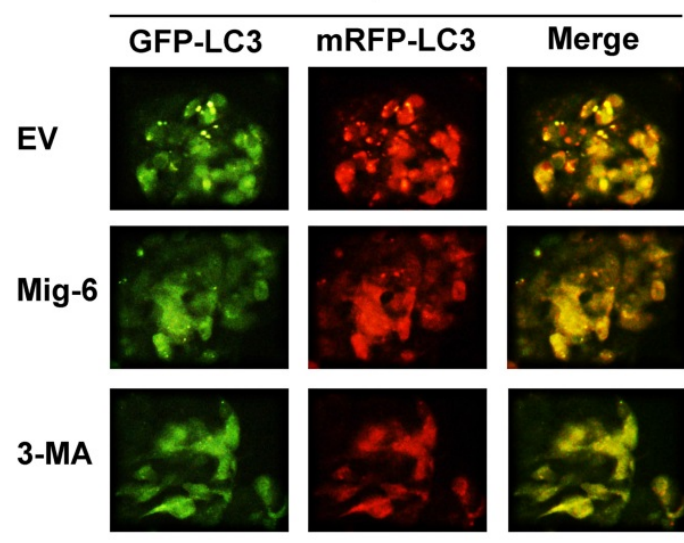

HepG-2

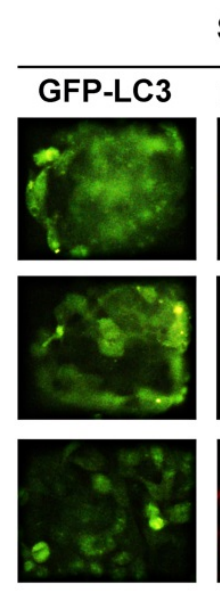

SMMC7721

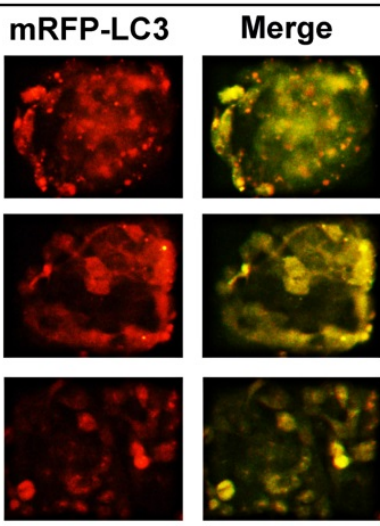

Figure 2. Mig-6 enhances autophagy in HCC cells. (A) Western bloting analysis showed that after Mig-6 transfection or added 3-MA in HepG-2 and SMMC7721 cell lines protein expression of LC3b were significantly decreased, but $\mathrm{p} 62$ were significantly increased. 3-MA was applied at $1.5 \mathrm{mg} / \mathrm{ml}$. (B) Each group was transfected with a tandem mRFP-GFP-LC3 adenovirus for 24 hrs. Autophagosomes and autolysosomes were, respectively, visualized as yellow- and red-only punctas under a fluorescence microscope. (C) Statistical analysis of fluorescent points in HCC cells.

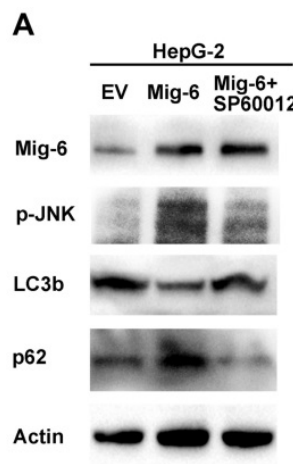

B

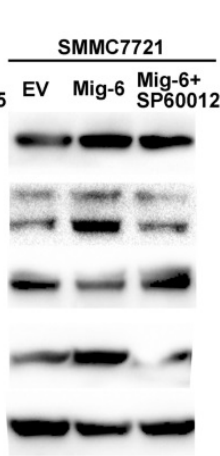

HepG-2
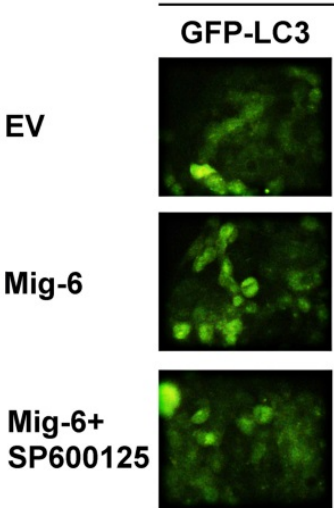

Mig-6

EV
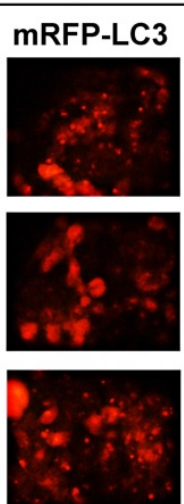
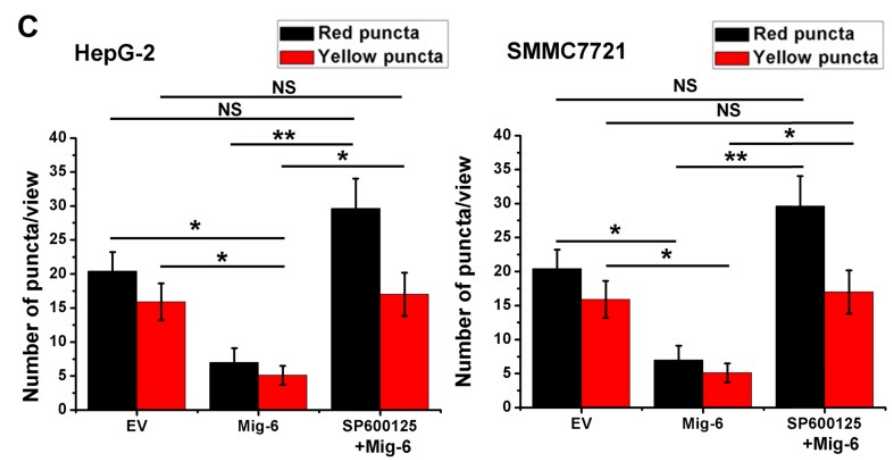

SMMC7721

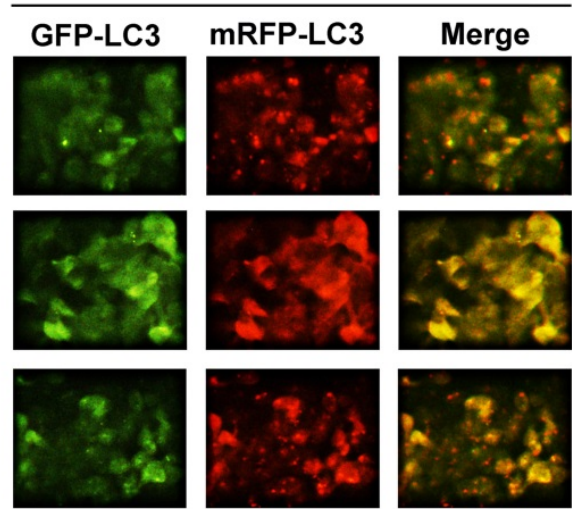

Figure 3. Mig-6 inhibits the autophagy of HCC cells by activating the SAPK / JNK pathway. (A)HepG-2 and SMMC7721 cell lines were transfected with Mig-6 for $24 \mathrm{~h}$, followed by treatment with SP600125(10 $\mu \mathrm{M}, 24 \mathrm{~h})$. Then the expression ofp-JNK,JNK,LC3b and p62 was detected by western bloting analysis. $\beta$-Actin served as a loading control.(B) Each group was transfected with a tandem mRFP-GFP-LC3 adenovirus for $24 \mathrm{~h}$. Autophagosomes and autolysosomes were, respectively, visualized as yellow- and red-only punctas under a fluorescence microscope. (c) Statistical analysis of fluorescent points in HCC cells. 
A

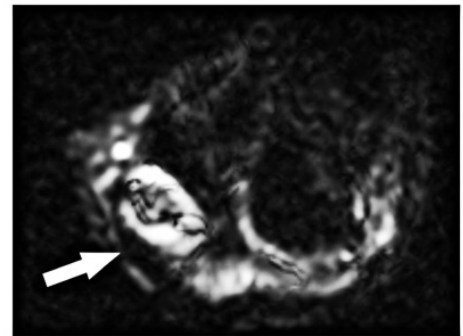

C

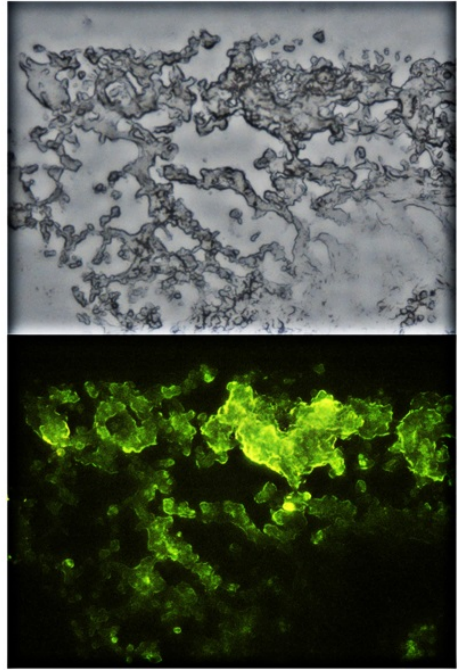

B

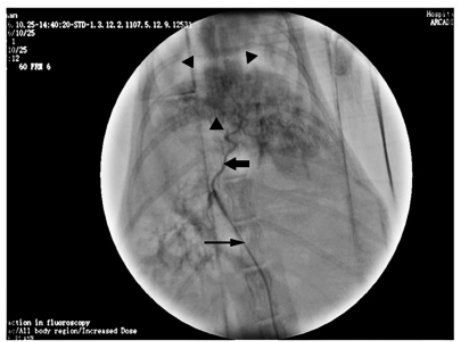

D

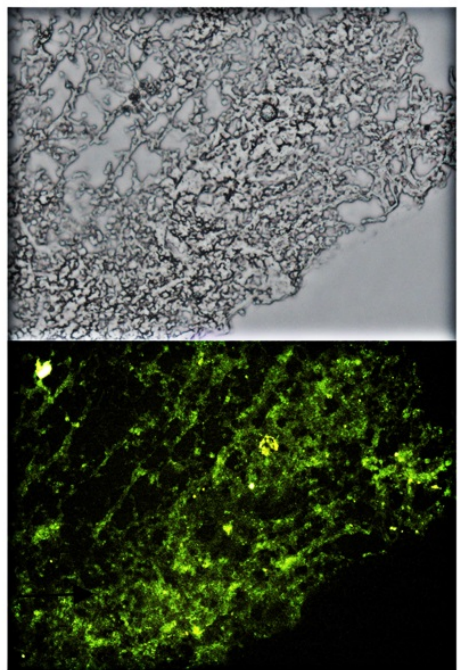

E

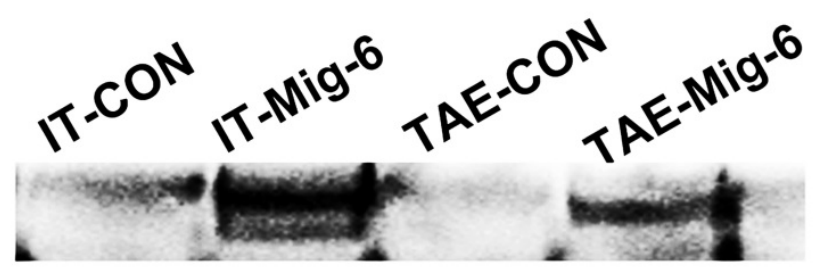

Actin

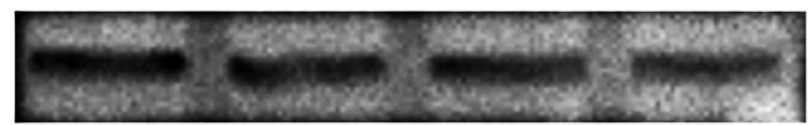

Figure 4. The establishment of the animal model and AAV9-mediated Mig-6-GFP expression in rabbit VX2 tumors. (A) Magnetic resonance imaging-based detection of tumor formation in the rabbit VX2 hepatocellular carcinoma model. ( $\longrightarrow$ ) (B) TAE procedure. The therapeutic agent was administered via a microcatheter ( ) inserted into the hepatic artery ( $\longrightarrow$ ), which can be seen feeding the tumor ( $)$ ). (C) Visualization of GFP expression in VX2 tissue after injection of AAV-CMV-Mig-6-GFP-3FLAG. (D) Visualization of GFP expression in VX2 tissue after AAV-CMV-Mig-6-GFP-3FLAG was delivered via arterial catheterization. (E) The expression of GFP-Mig-6 in each group on day 21 after operation.

\section{In vivo gene transfer in a rabbit $\mathrm{VX2}$ liver tumor model and assessment of Mig-6 expression.}

Fresh VX2 tumor fragments from the thigh muscles of a carrier rabbit were used for the tumor model and resulted in 100\% tumor formation (Fig. 4A).We chose rabbits with tumor diameters of 10-20 $\mathrm{mm}$ for direct intra-tumoral (IT) therapy or Transcatheter arterial embolism (TAE) with the help of digital subtraction angiography (DSA) (Fig.4B). Three weeks after AAV infection, all the animals were sacrificed with an overdose of Beuthanasia-D. Frozen sections were made from each tissue sample for fluorescence microscopy in order to determine transfection efficiency. Fluorescence microscopy demonstrated the expression of Mig-6-GFP on the tumor cell as expected (Fig. 4C,D). IT and TAE routes of AAV-CMV-Mig-6-GFP-3FLAG delivery resulted in similar expression of reporter gene. This was further confirmed by western blotting (Fig. 4E), which revealed clear and similar Mig-6-GFP bands after IT and TAE routes of AAV-CMV-Mig-6-GFP-3FLAG delivery.

\section{The efficacy of AAV-Mig-6 combined with TAE is superior to that of TAE alone.}

Tumor volumes were examined 21 days after TAE. In contrast to the similarlyized tumors observed before the treatments, tumor sizes on day 21 exhibited a wide range. The average size of tumors in the control group was $20.63 \pm 2.1 \mathrm{~cm}^{3}$, while in the 
TAE-alone group it was $12.33 \pm 3.2 \mathrm{~cm}^{3}$. In the AAV-Mig-6+TAE group tumor size had fallen to 5.83 $\pm 0.58 \mathrm{~cm}^{3}$ (Fig. $\left.5 \mathrm{~A}\right)$. With respect to growth rate, tumors in the AAV-Mig-6+TAE group showed a slight growth, mild growth was observed in response to TAE alone, while tumors in the control group had grown markedly (Fig.5B). The growth rate in the AAV-Mig- $6+\mathrm{TAE}$ group was $65.45 \% \pm 3.92 \%$, while in TAE it was $252.37 \% \pm 12.34 \%$. This difference is significant $(\mathrm{p}=0.001)$, which indicates an enhanced anti-tumor effect of AAV-Mig-6+TAE. In conclusion, Mig- 6 enhances the efficacy of TAE in the rabbit VX2 liver tumor model.

The safety of AAV was also assessed in the rabbit VX2 liver tumor model. A time course study of the plasma concentrations of alanine aminotransferase (ALT) and aspartate aminotransferase (AST) before and after the treatments is shown in Fig. 5C. At the outset of the experiment, levels of ALT and AST were similar to each other in the different groups. There were no significant changes in ALT and AST levels in the two IT groups, over the course of the two weeks of the experiment. In the two TAE groups, three days after treatment, ALT and AST levels increased transiently and significantly, but had decreased by day seven. On the 14th day, ALT and AST

A

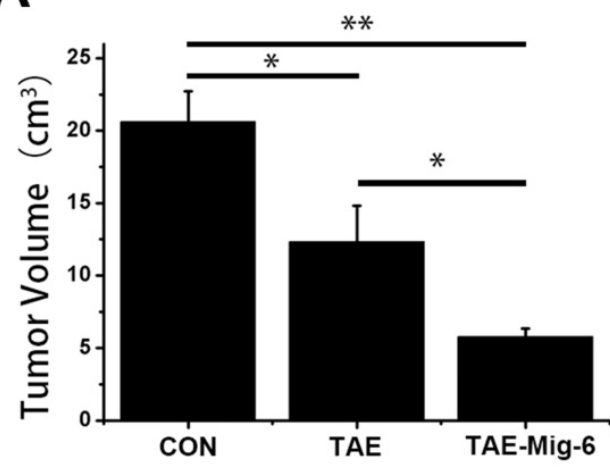

C

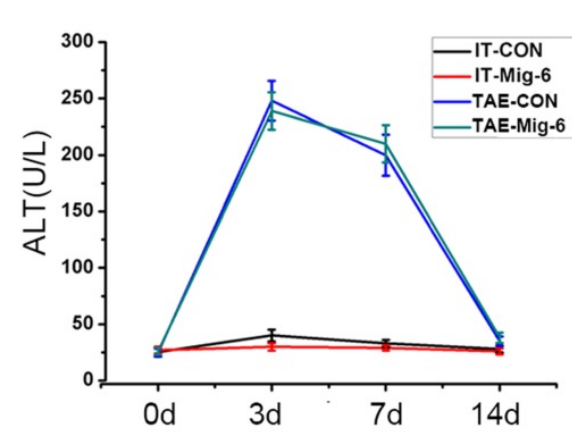

concentrations in the TAE and AAV-Mig-6+TAE groups were significantly higher than two IT groups. However, neither ALT nor AST in the AAV-Mig-6+TAE group were higher than in the TAE group. So, the AAV-Mig-6 plus TAE is as safe as TAE.

\section{Upregulation of Mig-6 expression activate p-JNK and inhibit the autophagy of liver tumors in VX2 tumors.}

Tumor samples were harvested from the solid part of the tumor, and necrotic areas were avoided. For the two experimental groups using AAV-Mig-6, the tissues that transfection efficiency has been verified by fluorescence microscopy were selected for further testing. Then, we examined the expression of p-JNK, LC3b and p62 in VX2 tumors. Western blotting revealed a significant increase in the expression of p-JNK in IT-Mig-6 and AAV-Mig-6+TAE groups. We also examined autophagy-related proteins and found that over-expression of Mig-6 inhibited LC3b expression but reduced p62 expression. Immunohistochemistry also revealed a significant increase in the expression of p-JNK and LC3b, and decrease in the expression of p62 (Fig. 6). The above results further validate our previous experimental results in liver cancer cells.

\section{B}
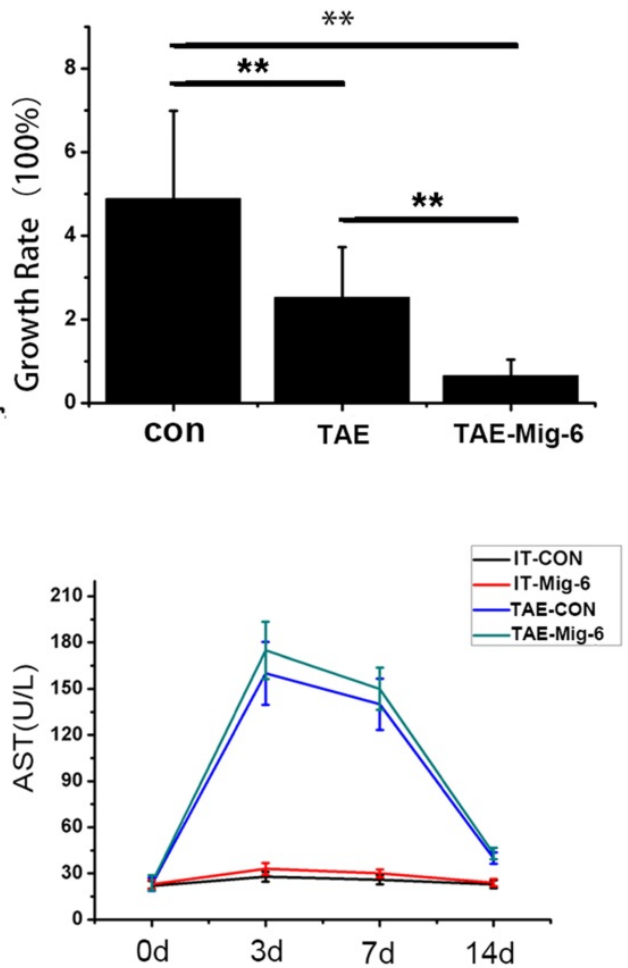

Figure 5. AAV-Mig-6 enhances the antitumor effect of TAE in vivo. (A) Tumor volumes after treatment with TAE and AAV-Mig-6+TAE were significantly smaller than in the control. The volumes in the AAV-Mig-6+TAE group were smaller than those in the TAE alone group. (B) The growth rate was calculated according to the change in tumor volumes. The values are shown as means \pm SD. Highly statistically significant differences are marked with $* *(p<0.01)$. (C)Concentrations of ALT and AST in the blood increased transiently at three days after TAE alone as well as after TAE in combination with AAV-Mig-6. At 14 days after TAE or AAV-Mig-6+TAE, the levels of both enzymes returned to pre-treatment levels in all groups. The values are shown as means \pm SD and the results show no statistically significant difference between TAE and AAV-Mig-6+TAEgroups. 
A

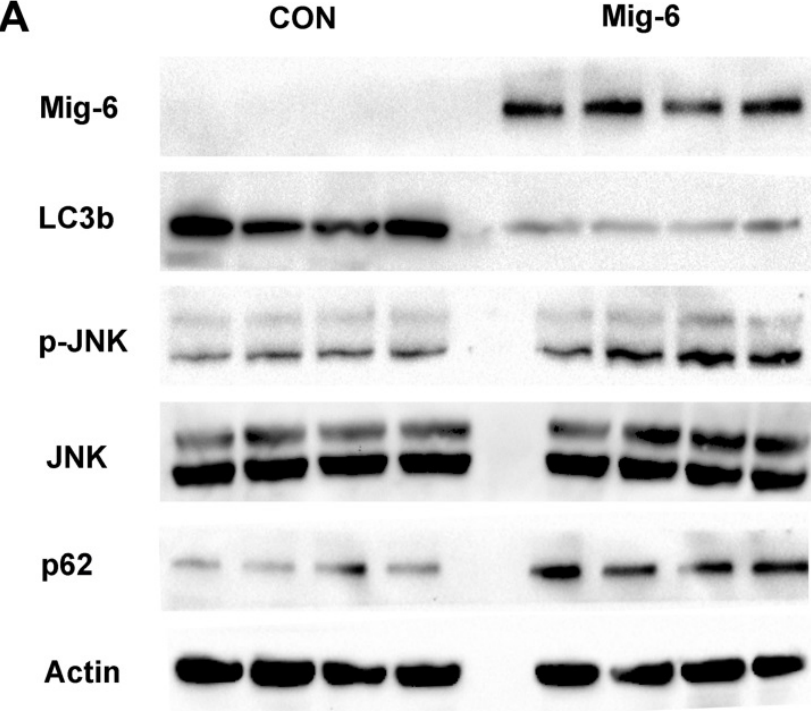

B

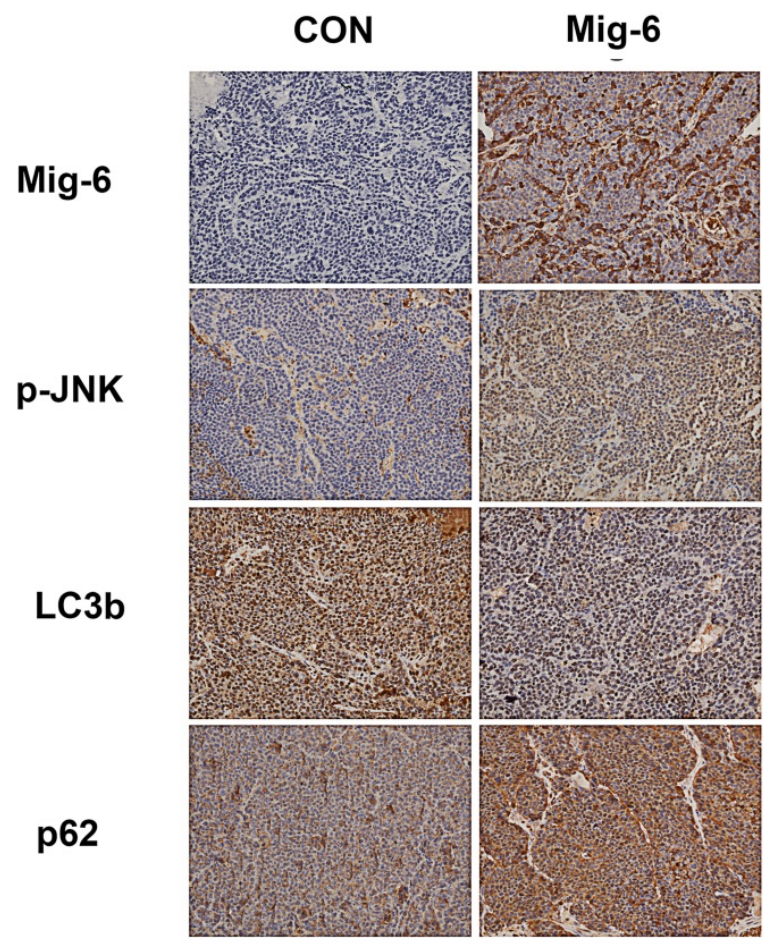

Figure 6. Upregulation of Mig-6 expression activate the SAPK/JNK signaling pathway and inhibit the autophagy in the rabbit VX2 hepatocellular carcinoma model. (A) Western blotting for Mig-6, p-JNK, JNK, LC3b and p62 expression in IT-control and IT- AAV-Mig-6 upregulation groups. (B) Western blotting for Mig-6, p-JNK, JNK, LC3b and p62 expression in TAE-control and AAV-Mig-6+TAE groups.(C) Immunohistochemistry for Mig-6, p-JNK, JNK, LC3b, p62,and Ki67 expression in all IT and TAE groups (200×).

\section{Discussion}

The adaptor protein Mig-6 is highly expressed in liver more than other organs such as fat, muscle, adrenal gland, and lung [9]. Liver-specific knockout mice of Mig-6 revealed hepatomegaly, hypercholesterolemia, fatty Liver and insulin resistance, suggesting that Mig-6 protein in the liver might play an important role in metabolism $[10,11]$.
Our previous studies demonstrated that Mig- 6 was downregulated in liver cancer and correlated with the prognosis. Mig-6 overexpression significantly inhibited cell viability of HCC cell lines. However, the underlying mechanisms of Mig- 6 in HCC are poorly understood.

Autophagy is an evolutionarily which degrades cell's components adapt to tough circumstances. More importantly, dysfunction of autophagy contributes to the pathologies of many human diseases, including HCC (hepatocellular carcinoma) [12]. Previous study found that combination of autophagic inhibitor and TACE may enhance the efficacy of TACE, in a rabbit VX2 liver tumor model. Therefore, in this study, we first examined whether Mig- 6 could affect autophagy in HCC cells. We found that up-regulation of Mig-6 expression in hepatocarcinoma cells can decreased LC3b, a marker of autophagy, but increased p62, an indicator of cytosolic protein clearance. Furthermore, red and green fluorescent dyes were used for the detection of LC3b, as observed through the lack of green and red fluorescence, it is suggested that up-regulation of Mig- 6 results in autophagy inhibition.

A number of studies have demonstrated the role of Mig-6 in the regulation of PI3K / AKT / mTOR pathway and ERK/MAPK pathway. Although both of these pathways have regulatory effects on autophagy in HCC [13]. But these results cannot explain the mechanism of Mig-6 inhibtion autophagy in liver cancer cells.

We analyzed the molecular structure of Mig-6 and found that its amino-terminal Cdc42/Rac interaction and binding (CRIB) domain can bind and activate CDC42 [14]. Human Cdc42 is a small GTPase of the Rho family, which regulates signaling pathways that control diverse cellular functions including cell morphology, cell migration, endocytosis and cell cycle progression [15]. Interestingly, another study confirmed that activated Cdc42 potently activate JNK in COS-7 cells [16]. To verify whether Mig- 6 could affect JNK activation, we up-regulated Mig-6 expression in HepG2 cells and found that up-regulation of p-JNK expression was detected. Several studies have investigated the effect of p-JNK on autophagy in tumor cells. Exacerbation of diabetic cardiac hypertrophy in mice is associated with JNK mediated autophagy inhibition [17]. Vitexin suppresses autophagy in hepatocellular carcinoma via activation of the JNK signaling pathway [18]. In this study we found up-regulation of Mig-6 significant increased p-JNK, and inhibited autophagy. If up-regulation of Mig-6 cotreatment with JNK inhibitor SP600125, the inhibition of autophagy by Mig- 6 was reversed. All these demonstrate the ability 
of Mig-6 inhibit autophagy in liver cancer cells is at least partially mediated through the activation of p-JNK.

Mig-6 knockout transgenic mice showed enhanced liver regeneration ability compared to wild-type mice, suggesting an important role for Mig-6 in influencing cell proliferation [19].

However, studies describing the upregulation of Mig-6 expression in tumor tissue are still missing. We used a rabbit liver cancer model to investigate the effects of up-regulation of Mig-6 on the microenvironment of liver cancer after TAE. Rabbits were treated with transarterial injection of AAV-Mig-6 in combination with TAE. TAE plus chemotherapy drugs is a commonly used method in the clinical treatment of HCC. In order to exclude the effect of chemotherapeutic agents on the effect of Mig-6, we used TAE as a treatment in this experiment. Compared with other systemic methods of gene therapy, this method ensures that the expression of the delivered gene is more strictly confined to the target organ. After two weeks of treatment, AAV-Mig- 6 perfused by arterial catheterization was able to achieve similar transfection efficacy as direct tumor injection.The TAE group and the AAV-Mig-6+TAE group both exhibited an inhibitionof tumor growth. However, the AAV-Mig-6+TAE treatment was more effective in the inhibition of tumor growth than the TAE alone group. This demonstrates that AAV-Mig-6 functions to promote the efficacy of traditional TAE.

A previous study showed that a transient increase in mean ALT levels was observed in the high-dose scAAV2/8-LP1-hFIXco gene therapy group $[20,21]$. In this study, we found that injection of AAV-Mig-6 alone had little effect on liver function. ALT and AST concentrations in the TAE and AAV-Mig-6+TAE groups were significantly higher than in the control after the treatments. However, these values were not higher in AAV-Mig-6+TAE groups than in the TAE alone group. These data show that the combination of AAV-Mig-6 and TAE can inhibit tumor growth substantially without severe adverse effects on background liver function, at least in the first two weeks. Since we did not make observations over a longer period of time, further experiments are needed to verify the long-term biosafety of AAV together with TAE.

In order to observe the effect of up-regulating Mig- 6 on tumor microenvironment after TAE, we examined the expression of autophagy-related genes in the tissues transfected with Mig- 6 . We found that the expression of LC3b decreased after upregulation of Mig-6, but the p62 increased. The up-regulation of
p-JNK expression was also detected which was consistent with in vitro results.

These results suggest that the effect of Mig- 6 transfection on TAE may be achieved in part by inhibiting the autophagy through activated JNK pathway.

Much research is currently focused on the combination of TACE with other drugs, in order to improve the efficacy of the TACE. Several reaserch found that gene therapy in combination with TACE is superior to TACE alone in terms of the survival rate and improved symptoms of HCC patients [22, 23]. Until now, the TACE vectors that have been combined with gene therapy we are all adenovirus-derived. However, adenoviral vectors are known to support only transient expression lasting approximately one week, which is a major drawback that limits their application. This short persistence is due to the mounting of a potent immune response against the virus [24].

Wild-type AAV is not associated with any human or animal disease, has very low immunogenicity and has been used in human gene therapy in 92 clinical trials [6]. There are over 100 different AAV serotypes that vary in the amino acid sequence oftheir capsid protein. However, so far, no AAV serotype has been used to deliver genesfor transfection in the liver transplant tumor model. In this study, we injected AAV9 encoding Mig-6 into the VX2 liver transplant tumors of rabbits by direct injection and TAE. The results showed that both methods of administration resulted in high transfection efficiency.

There were several limitations to our study. First, to clarify the function of Mig-6 in HCC, we perfused AAV-Mig-6 via the hepatic artery without any chemotherapeutic agents. This is inconsistent with the commonly used clinical treatment. Second, in vivo experiments were performed in a rabbit model of HCC, which may limit the extrapolation of the results to human HCCs.

Finally, only a preliminary discussion had been made of Mig-6 inhibition of autophagy in HCC. More research is needed to refine our understanding of the role of Mig-6 in the regulation of autophagy.

In summary, this study for the first time using AAV mediates tumor suppressor gene expression through hepatic artery perfusion in liver cancer. It will provide important reference value for future research on hepatocellular carcinoma in vivo and research on tumor biological therapy. In addition, up-regulating the expression of Mig-6 in vivo and exploring the mechanism of its regulation of autophagy are of great significance for our understanding the function of Mig-6 in tumorigenesis. 


\section{Acknowledgements}

This work was supported by grants from the National Natural Science Foundation of China (No. 81501564).

\section{Competing Interests}

The authors have declared that no competing interest exists.

\section{References}

1. Torre LA, Bray F, Siegel RL, Ferlay J, Lortet-Tieulent J, Jemal A. Global cancer statistics, 2012. CA: a cancer journal for clinicians. 2015; 65: 87-108.

2. Hanahan D, Weinberg RA. Hallmarks of cancer: the next generation. Cell. 2011; 144: 646-74.

3. Huang $\mathrm{P}, \mathrm{Xu} \mathrm{X}$, Wang L, Zhu B, Wang $X$, Xia J. The role of EGF-EGFR signalling pathway in hepatocellular carcinoma inflammatory microenvironment. Journal of cellular and molecular medicine. 2014; 18: 218-30

4. Kunimoto T, Baba H, Nitta K. Rapid tumor regression caused by antitumor polysaccharide and induction of tumor-regressing factor in the serum of tumor-bearing mice. Journal of biological response modifiers. 1986; 5: 160-7.

5. Gao L, Song JR, Zhang JW, Zhao X, Zhao QD, Sun K, et al. Chloroquine promotes the anticancer effect of TACE in a rabbit VX2 liver tumor model. International journal of biological sciences. 2013; 9: 322-30.

6. Ginn SL, Alexander IE, Edelstein ML, Abedi MR, Wixon J. Gene therapy clinical trials worldwide to 2012 - an update. The journal of gene medicine. 2013; 15: 65-77.

7. Gao G, Vandenberghe LH, Alvira MR, Lu Y, Calcedo R, Zhou X, et al. Clades of Adeno-associated viruses are widely disseminated in human tissues. Journal of virology. 2004; 78: 6381-8.

8. Bruix J, Reig M, Sherman M. Evidence-Based Diagnosis, Staging, and Treatment of Patients With Hepatocellular Carcinoma. Gastroenterology. 2016; 150: 835-53.

9. Mirsalis JC, Provost GS, Matthews CD, Hamner RT, Schindler JE, O'Loughlin $\mathrm{KG}$, et al. Induction of hepatic mutations in lacI transgenic mice. Mutagenesis. 1993; 8: 265-71.

10. Ku BJ, Kim TH, Lee JH, Buras ED, White LD, Stevens RD, et al. Mig-6 plays a critical role in the regulation of cholesterol homeostasis and bile acid synthesis. PloS one. 2012; 7: e42915.

11. Park BK, Lee EA, Kim HY, Lee JC, Kim KS, Jeong WH, et al. Fatty Liver and Insulin Resistance in the Liver-Specific Knockout Mice of Mitogen Inducible Gene-6. Journal of diabetes research. 2016; 2016: 1632061.

12. Gozuacik D, Kimchi A. Autophagy as a cell death and tumor suppressor mechanism. Oncogene. 2004; 23: 2891-906.

13. Liu L, Liao JZ, He XX, Li PY. The role of autophagy in hepatocellular carcinoma: friend or foe. Oncotarget. 2017; 8: 57707-22.

14. Makkinje A, Quinn DA, Chen A, Cadilla CL, Force T, Bonventre JV, et al. Gene 33/Mig-6, a transcriptionally inducible adapter protein that binds GTP-Cdc42 and activates SAPK/JNK. A potential marker transcript for chronic pathologic conditions, such as diabetic nephropathy. Possible role in the response to persistent stress. The Journal of biological chemistry. 2000; 275: 17838-47.

15. Qadir MI, Parveen A, Ali M. Cdc42: Role in Cancer Management. Chemical biology \& drug design. 2015; 86: 432-9.

16. Coso OA, Chiariello $\mathrm{M}, \mathrm{Yu}$ JC, Teramoto $\mathrm{H}$, Crespo $\mathrm{P}, \mathrm{Xu} \mathrm{N}$, et al. The small GTP-binding proteins Rac1 and Cdc42 regulate the activity of the JNK/SAPK signaling pathway. Cell. 1995; 81: 1137-46.

17. Oian LB, Jiang SZ, Tang XQ, Zhang J, Liang YQ, Yu HT, et al. Exacerbation of diabetic cardiac hypertrophy in OVE26 mice by angiotensin II is associated with JNK/c-Jun/miR-221-mediated autophagy inhibition. Oncotarget. 2017; 8: 106661-71.

18. He JD, Wang $\mathrm{Z}, \mathrm{Li}$ SP, $\mathrm{Xu}$ YJ, $\mathrm{Yu} \mathrm{Y}$, Ding $\mathrm{YJ}$, et al. Vitexin suppresses autophagy to induce apoptosis in hepatocellular carcinoma via activation of the JNK signaling pathway. Oncotarget. 2016; 7: 84520-32.

19. Reschke M, Ferby I, Stepniak E, Seitzer N, Horst D, Wagner EF, et al. Mitogen-inducible gene- 6 is a negative regulator of epidermal growth factor receptor signaling in hepatocytes and human hepatocellular carcinoma. Hepatology. 2010; 51: 1383-90.

20. Nathwani AC, Tuddenham EG, Rangarajan S, Rosales C, McIntosh J, Linch $\mathrm{DC}$, et al. Adenovirus-associated virus vector-mediated gene transfer in hemophilia B. The New England journal of medicine. 2011; 365: 2357-65.

21. Nathwani AC, Reiss UM, Tuddenham EG, Rosales C, Chowdary P, McIntosh $\mathrm{J}$, et al. Long-term safety and efficacy of factor IX gene therapy in hemophilia B. The New England journal of medicine. 2014; 371: 1994-2004.

22. Guan YS, Liu Y, He Q, Li X, Yang L, Hu Y, et al. p53 gene therapy in combination with transcatheter arterial chemoembolization for HCC: one-year follow-up. World journal of gastroenterology. 2011; 17: 2143-9.

23. Lin XJ, Li QJ, Lao XM, Yang H, Li SP. Transarterial injection of recombinant human type-5 adenovirus $\mathrm{H} 101$ in combination with transarterial chemoembolization (TACE) improves overall and progressive-free survival in unresectable hepatocellular carcinoma (HCC). BMC cancer. 2015; 15: 707.

24. Yang Y, Nunes FA, Berencsi K, Furth EE, Gonczol E, Wilson JM. Cellular immunity to viral antigens limits E1-deleted adenoviruses for gene therapy. Proceedings of the National Academy of Sciences of the United States of America. 1994; 91: 4407-11. 